\title{
On the Possible Deeper Structure of Leptons and Quarks: A View of the "Ultron"-“Logotron" Theory
}

\author{
Sung Jang Chung \\ Morristown-Hamblen Healthcare System, Morristown, USA \\ Email: sung.chung@comcast.net
}

Received 24 March 2015; accepted 22 May 2015; published 25 May 2015

Copyright (C) 2015 by author and Scientific Research Publishing Inc.

This work is licensed under the Creative Commons Attribution International License (CC BY). http://creativecommons.org/licenses/by/4.0/

(c) (i) Open Access

\begin{abstract}
The deeper structure of leptons and quarks is still not experimentally and/or objectively clearly elucidated. No detailed accepted theory is yet established. Consequently, experimental and theoretical physicists have been investigating to find experimental or objective proofs related to the deeper structure of fundamental elementary particles in quantum physics. The author proposed a hypothesis of the "ultron"-logotrn" theory that might explain relationships between self and consciousness: mind and matter on the basis of his review of modern quantum physics and his personal experience of valid precognitive dreams, and further published an article that describes a considerable parallels between Confucian philosophy and quantum physics from the ontological perspective. Ultrons are the building blocks of matter of the universe. Logotrons are the building blocks of conscious mind. In this study, possible deeper structures of leptons and quarks: yin- and yang-ultron with mass of $0.16 \mathrm{eV}$, electric charge of - and $+1.602176565 \times 10^{-19}$ coulomb/ $(3.19 \times$ $10^{6}-2 k$ ) (see Equations (7) and (8)), size less than $1.616199 \times 10^{-35} \mathrm{~m}$, total number of ultrons as well as number of yin- and yang-ultrons in a neutrino, electron, positron; and up, down, strange quark (see Equations (2) to (6) and (9)) are presented on the basis of reported data in particle physics and the perspective of the author's hypothesis of the "ultron"-“logotron" theory.
\end{abstract}

\section{Keywords}

Leptons, Quarks, Neutrinos, Quantum Physics, Ultron-Logoton Theory, Confucianism, I Ching, Jeong Yeok, Theory of Everything, Probacent Model, Precognitive Dream

\section{Introduction}

The deeper structure of leptons and quarks is still not experimentally or objectively clearly elucidated. Accord- 
ing to Tong (2009), theoretical physicist, "there is no experimental evidence that string theory is the correct description of our world”. No detailed accepted theory is yet established (Caesar, 2014). Consequently, experimental and theoretical physicists have been investigating to find experimental and/or objective proofs related to the deeper structure of fundamental elementary particles in quantum physics.

\subsection{The Science of Self, Mind and Body}

The human self is the agent being the source of consciousness and responsible for the thoughts of mind and actions of body in an individual, enduring through the time.

The author had unusual personal experiences that difficult mathematical problems were not solved in his manifest consciousness but could be unexpectedly clearly solved in his subconscious mind (Chung, 2009). The author published the solution of the math problem in the International Journal of Biomedical Computing (Chung, 1995). The math problem was regarding a general mathematical model of "probacent”-probability equation that expresses a relationship among intensity of stimulus, duration of exposure and occurrence of response in biological phenomena (Chung, 1960, 2007, 2013). The mathematical model might possibly calculate the probability of safe survival in humans and other living organisms exposed to any harmful or adverse circumstances or conditions, overcoming the risk.

The author proposed a theory that there are in human individuals two selves, one, the inner self (the true self, spirit) and one, the physical self (the false self) that coexist in one individual person (Chung, 2009, 2012). McGonigal (2012) describes that one individual has two minds or two selves, naming “I WILL” and “I WANT” selves.

More recent researches in neuroscience using functional magnetic resonance imaging (fMRI) discovered that the prefrontal cortex (PFC) of the human brain performs cognitive control, emotion regulation, moral self-control, control of impulsive behavior and guiding brain activities with future goals and rules (Miller \& Cohen, 2001; Greene et al., 2001; Ochsner et al., 2002; Schwartz, 1999).

The author (Chung, 2012) published a theory regarding the scientific relationship among self, mind and body based on the above described findings that a human individual, the self is composed of the inner true self (spirit) and the physical false self that would fade and disappear at death of the body. The inner self has free will, will power, cognition, reason, morality, conscience, creative power, mathematical computation, future plan and high goals, behavior control, emotion regulation, and memory retrieval, supervising the physical self/body that senses through sensory organ systems and responds to the external world. The inner true self is the independent and indestructible spirit in an individual. The physical self is dependent upon and associated with the brain and body, and is impulsive, behaving for pleasure-desire and instinct for living. The inner self controls the physical self/ body by interacting with the prefrontal cortex of human brain.

Scientific relationships and interactions between self and consciousness: mind and matter in the neurons of the prefrontal cortex are not clearly understood (Penrose, 2007; Penrose, Hameroff, \& Kak, 2011; Penrose \& Hameroff, 2011; Gusnard, 2009; Bohm, 2006).

\subsection{Quantum Physics}

All physical processes in the physical world are based on particles and forces (Hawking, 1996; Best, 2015). All particles are in facts waves (Hawking, 1996, 2001).

A wave-particle duality of electron proved by Thomas Young and Richard Feynman's double-slit experiments has not been explained (Aczel, 2003; Nichol, 2006). According to Bohm (2006), this phenomenon suggests that an electron seems to have consciousness, interacting with the environmental background field of space. This is a transformation for a wave to a particle, and vice versa like an organism than interacting parts of an inanimate machine (Nichol, 2006).

Matter, energy and mind are different aspects of the unknown, unbroken flowing movement of totality, the wholeness. Consciousness may be the essential nature of the universe and a more subtle form than matter of the universe (Bohm, 2006). The totality, the wholeness is nonlocal and timeless. Contents of consciousness are called by Bohm “information”. The author names “information” “logotron”, 言子 in this study (Greek: logos).

\subsection{Precognitive Dreams}

The author had unusual precognitive dreams during the World War II and the Korean War that saved not only 
the author but also other people (Gruenberg, 1959; Chung, 2009, 2012, 2014a). Information received in the precognitive dreams of future events that took place in reality was verified as true (Chung, 2014a). In the history of the United States, one of the great presidents, Abraham Lincoln had dreams of his death 13 days before he was assassinated (Joseph, 2011a). According to Joseph (2011a), certain cognitive capacities are well developed in just a few people. Precognitions of future events are well described in religions (the Bible, the Buddhist and Hindu scriptures). The author's personal experiences of precognitive dreams that are verified as true seem to strongly suggest the work of the author's inner self and further to reveal a glimpse into an unknown, timeless and nonlocal consciousness world of reality, i.e. the invisible spiritual world (Chung, 2009, 2012, 2014a).

\subsection{Quantum Entanglement}

Chien-Shiung Wu and Irving Shaknov in 1949 produced positronium that released two photons. David Bohm and Yakiv Aharonov in 1957 first recognized quantum entanglement in their analysis of results of Wu and Shaknov's experiment. John Clauser and Stuart Freedman in 1972 produced experimental evidences of entanglement (Aczel, 2003).

Alain Aspect and his collaborators in 1980s, Nicholas Gisin in 1990s and Andrew Watson in 1997 clearly observed in their experiments that the speed of transmission of mutual effect between two entangled particles was faster than the speed of light (Aczel, 2003; Penrose, Hameroff, \& Kak, 2011; Laszlo, 2007; Watson, 1997). The instantaneous influence between two photons or electron twins with same quantum states violates the speed of light according to Einstein's theory of relativity. The phenomenon of entanglement proves that the EPR paradox theory of locality is incorrect and quantum theory of non-locality is correct (John Bell's theorem) (Griffiths, 2005; Aczel, 2003). The EPR paradox theory of Einstein, Podolsky and Rosen holds the principle of locality that what happens in one local place does not immediately affects what happens in another local place in space. The EPR theory objects to instantaneous faster-than-light transmission of physical effects between two objects such as photons and electrons at a distance ("spooky action at a distance") that can be observed in entanglement and non-locality in quantum physics.

Penrose and Hameroff (2011) and Hameroff (2013) proposed the Orch OR theory with microtubule quantum computations to explain interactions between mind and matter within neurons of brain.

Eugene Wigner proposed a hypothesis that conscious intervention of an observer can cause the collapse of the quantum wave function and this determines contents of the brain (Stapp, 2011). In John von Neumann's theory of emergent dualism, some conscious agent intervenes upon the state of the brain and does conscious choice, controlling the mind-body connection in the brain (Stapp, 1999, 2011). The von Neumann-Heisenberg orthodox quantum mechanics supports the dualism in quantum physics. The author infers that the agent seems to be the inner self of human being.

Interactions between self and consciousness: mind and matter are not clearly understood in science. There seem to be, to my knowledge, no articles in the literature that clearly explain the relationship between self and consciousness: mind and matter. The relationship between self and consciousness: mind and matter is investigated and a hypothesis is developed to explain it and observations.

\subsection{Self and Consciousness: Mind and Matter (The Ultron-Logotron Theory)}

Interactions between self and consciousness: mind and matter are not clearly understood in science. There seem to be, to my knowledge, no articles in the literature that clearly explain the relationship between self and consciousness: mind and matter.

In the study, the relationship is investigated and an attempt is made to explain it.

1) Findings and discoveries in modern science, quantum physics, string theory, especially entanglement in the arena of mind and matter are reviewed (Penrose, 2007; Penrose, Hameroff, \& Kak, 2011; Griffiths, 2005; Stapp, 1999, 2011; Tong, 2009; Ochsner et al., 2002; Schwartz et al., 2005; Mitchell \& Staretz, 2011; Close, 2011; Laszlo, 2007, 2008; Suplee, 1999; Riordan, 1987; Capra, 1999).

2) Philosophical teachings of major religions, especially the Eastern Confucian philosophy are reviewed and compared with quantum physics. Confucian philosophy is described in the I Ching, the Book of Changes (William, 1967) and Jeong Yeok, the Book of Right Change (Kim, 1885; Yi, 1992; Chung, 2009, 2010).

3) Review of some of relevant personal experiences of precognitive dreams of the author is included in the study. 
Comparison of characteristic aspects of the author's proposed theory of ultron-logotron and quantum physics is done. Interpretation, theoretical reasoning and imagination are carried out to reasonably explain observed findings, precognitive dreams, entanglement, and proposed theories regarding the scientific relationship between self and consciousness: mind and matter.

\subsection{1. "Ultron", 極子}

In Confucian philosophy, all things are created by power of the Creator, the Non-Ultimate, the Great Ultimate. The Great Ultimate created Two Forms, 兩儀, Yin and Yang, the two primary fundamental elementary particles that are represented by a solid line $(-)$ and a broken line $(--)$. The author names Yin and Yang "yin-ultron”, 陰極子 $(U)$, and “yang-ultron”, 陽極子 $\left(U^{+}\right)$, respectively. All things of the universe are generated by movements of "ultrons”, 極子 (Capra, 1999; Chung, 2009).

Table 1 shows comparison of characteristic aspects of the ultron-logotron theory and quantum physics. Basic elementary particles, ultrons, 極子 would be analogous to strings of string theory. The ultrons are the building blocks of matter of the physical world. The yang- and yin-ultrons are positively and negatively charged, respectively.

Findings in quantum physics are postulated to be applicable to the ultron.

The ultron is postulated to have a property of particle-wave duality.

The electric charge, $e_{\text {yin }}$ of yin-ultron may be $-1 e<e_{\text {yin }}<0$.

The electric charge of yang-ultron, $e_{\text {yang }}$ may be $0<e_{\text {yang }}<+1 e$.

The ultron mass, $m_{\text {ultron }}$ may be $0<m_{\text {ultron }}<$ mass of electron.

If external force comes to a region of space, the regional space will be excited and pulsate, and then a hidden vertical pair of particle-antiparticle emerges in it, causing material actualization of real particle and antiparticle, creating matter.

The author infers that when a matter and antimatter collide and disappear, becoming virtual matter and antimatter, consciousness in the excited state superpositioning to each of the pair will disappear and become a virtual consciousness in the ground state. If the above is true, then it would imply that particles of matter would have a triple property of particle, wave and consciousness (Bohm, 2006).

If a mental force is presumably created by the self, it will strike the regional space that could excite virtual consciousness in the ground state to become consciousness in the excited state, creating conscious mind (Chung, 2009). Mind and matter are interrelated and interconnected (Capra, 1999; Chung, 2009, 2012).

\subsection{2. "Logotron", 言子}

Table 2 shows comparison of characteristic aspects of "ultrons" and "logotrons". The author names "information” that is described by David Bohm in his quantum theory "logotron”, 言子. Logotrons in the study represent contents of consciousness of mind in humans such as thoughts, feelings, colors, sounds, concepts of all things of the universe that may be expressed by words (Greek: logos).

Logotrons are virtual particles, massless and have probably a triple property of particle, wave and consciousness, and have possessing inherent force, and having a property of spreading in space as well as remaining at an original, regional point in the holographic space. The wave propagation, if emitted from the logotron is postulated instantaneous in the non-local holographic space.

Table 1. Comparison of characteristic aspects of the "ultron”-“logotron” theory and quantum physics.

\begin{tabular}{|c|c|c|}
\hline Characteristic aspects & The "ultron"-“logotron" theory & Quantum physics \\
\hline Basic elementary particle & Yin- and yang-ultrons & Quarks and electrons \\
\hline Form & A solid $(-)$ and a broken $(--)$ line & An open $(\sim)$ and a closed $(0)$ string \\
\hline Movement & $\begin{array}{l}\text { Join, movement, stillness, advance, } \\
\text { retreat, expansion and contraction }\end{array}$ & $\begin{array}{l}\text { Split, fission, break, pinch, } \\
\text { join, spin and oscillation }\end{array}$ \\
\hline Property & Triple: particle, wave and consciousness & Triple: particle, wave and consciousness \\
\hline Energy & Yes & Yes \\
\hline Force & Yes & Yes \\
\hline
\end{tabular}


Table 2. Comparison of postulated characteristic aspects of "ultron” and "logotron”.

\begin{tabular}{|c|c|c|}
\hline Characteristic aspects & Ultron & Logotron \\
\hline Element of & Physical matter & Conscious mind \\
\hline Nature & Physical & Conscious (spiritual) \\
\hline Creation & Created by the creator & $\begin{array}{l}\text { Created by the creator and } \\
\text { co-creator (man) }\end{array}$ \\
\hline Property & Triple: particle, wave and consciousness & Triple: particle, wave and consciousness \\
\hline State & Real and virtual & Virtual \\
\hline Interaction between themselves & $\begin{array}{l}\text { Graviton, electromagnetic force, } \\
\text { weak and strong nuclear forces }\end{array}$ & Mental-force-carrying mentalon \\
\hline (Speed of transmission) & (Maximum speed of light) & (Instantaneous) \\
\hline
\end{tabular}

The empty space is postulated to be not void but filled with invisible and intangible energy and force, virtual ultrons and logotrons (virtual matter and consciousness), and further infinite power and love of the Creator God. It is said in the Bible (John 1:1-3 King James Version), "In the beginning was the Word, and the Word was with God, and the Word was God. The same was in the beginning with God. All things were made by him, and without him was not made any thing made that was made.” There seems to be an agreement between the Bible and the ultron-logotron theory.

\subsubsection{Interactions between Ultron and Logotron}

Figure 1 illustrates Feynman's space-time diagram of interactions between ultron and logotron. Mental-forcecarrying "mentalon” exchanges between ultron and logotron, creating resonance-like effects, and perhaps exchanges between logotrons as graviton exchanges between ultrons of matter. The conscious mind of the inner self of an individual person has power to generate mentalon as well as logotrons with free will and free choice. Mentalon is virtual force like graviton; it has two states: the ground and excited states. The author infers that the quantum states result from the incoming neural stimulus in the neurons of human brain, then OR occurs (the quantum wave function collapses) when the physical self and the inner self have conscious perception through quantum computations in the microtubule automata (Penrose \& Hameroff, 2011). In the quantum state of the prefrontal cortex of human brain, the quantum wave function collapses when the inner self who is an observer and does conscious cognitive action with free will and free choice, and with attention, efforts, thoughts and planning in the inner self's mind; then logotrons in the excited state are generated from the ground state, generating conscious cognition in the inner self's consciousness (Figure 1(a)) (Stapp, 1999; Kafatos, Tanzi, \& Chopra, 2011; Schwartz et al., 2005; Ochsner et al., 2002). The self (the true inner self) has power in the conscious mind to choose logotron in the excited state from its ground state, changing the quantum state of the logotron with free will, free choice, attention and intention, guiding actions (Stapp, 1999; Chung, 2012). Ultrons in the excited state are generated by mentalon originated from the inner self's conscious mind (Figure 1(b)) (Schwartz et al., 2005; Ochsner et al., 2002). Figure 1(b) illustrates processes at the interface between mind/ consciousness and brain. Existence of mentalons can be empirically recognized by their effects in the external world resulting from interactions between mind and matter in the PFC of human brain. In this matter-mind process, mentalons participate in generation of conscious mind (Figure 1(a)) and vice versa (Figure 1(b)). M line shows its origin on the left side with an apparent slightly upward right direction but is actually horizontal and parallel to the space axis, indicating instantaneous speed in space-time (Figure 1(a) and Figure 1(b)) (Penrose, Hameroff, \& Kak, 2011).

The author reviewed modern quantum physics and the Eastern Confucian philosophy. On the basis of the review and authors' personal experiences of valid precognitive dreams, the following theory is proposed (Chung, 2014a):

1) Valid and undeniable precognitive dreams of the author's personal experience and quantum entanglement strongly suggest the inner self's potential power transcending the time phases of the past, present and future, and existence of a timeless, nonlocal and holographic consciousness world of reality.

2) The ultrons" are the building blocks of matter of the universe. The "logotrons" are the building blocks of consciousness of human mind. The "logotrons" are virtual particles. The "ultrons" and "logotrons" interact each 


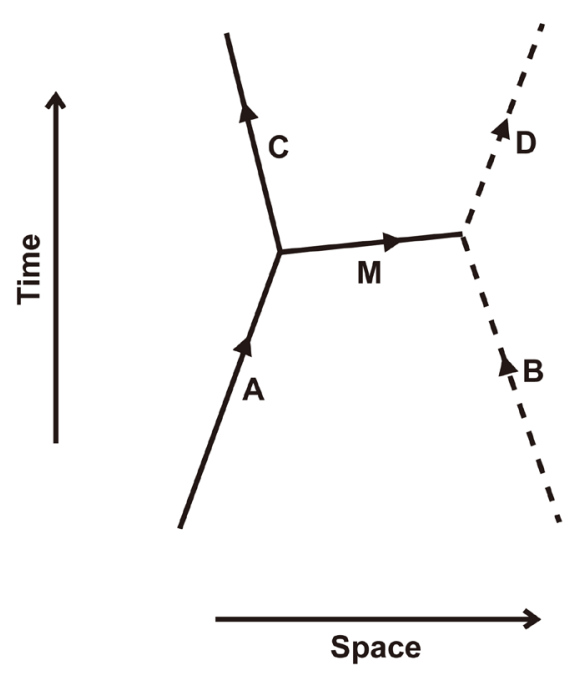

(a)

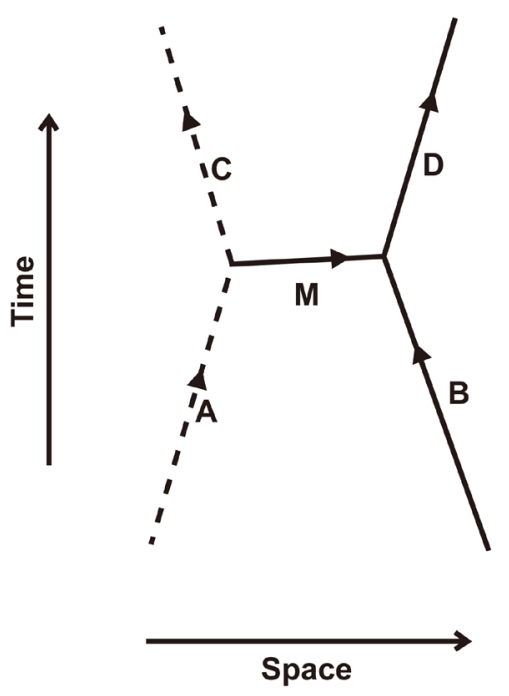

(b)

\begin{abstract}
Figure 1. Feynman's spacetime diagram of interactions between "ultron" and "logotron”. (a) A-Real ultron in the excited state in the neuron of the prefrontal cortex caused by incoming stimulus. C-Real ultron in the ground state after collapse of the quantum wave function when the self controls (observes). B-Virtual logotron in the ground state in the neuron of the prefrontal cortex. D-Virtual logotron in the excited state when the self observes and cognizes. M-Mental-force-carrying "mentalon" exchanging between ultron and logotron; (b) A-Virtual logotron in the excited state in the self's conscious mind in the neuron of the prefrontal cortex. C-Virtual logotron in the ground state after exchanging "mentalon" between the logotron and ultron in the neuron. B-Real ultron in the ground state in the neuron of the prefrontal cortex. D-Real ultron in the excited state in the neuron after exchanging "mentalon" between logotron and ultron. M-“mentalon".
\end{abstract}

other with mental-force-carrying "mentalon" in neurons of human brain. Mentalon exchanges between logotron and logotron or logotron of self's consciousness and logotron of consciousness superpositioned to particles of matter.

3) Entanglement could be explained by mental-force-carrying mentalon that exchanges between two entangled virtual quantum logotrons in particles or logotron in conscious mind and logotron in particles of matter at quantum levels.

4) Valid and undeniable precognitive dreams and experimentally accepted entanglement in quatum physics seem to provide concrete human data that might possibly explain a scientific relationship between self and consciousness: mind and matter.

5) There seem to be parallels between the "ultron"-“logotron” theory and quantum physics from the ontological perspectives, and a close agreement between the "ultron"-"logotron" theory and the Penrose-Hameroff 's Orch OR theory or the von Neumann-Heisenberg's orthodox quantum mechanics that seem to be correct descriptions and applicable to both the inner self and the physical self/body of humanity, respectively on the basis of the human individual self composed of two selves, one, the inner self and one, the physical self.

\title{
1.6. Parallels between Confucian Philosophy and Quantum Physics
}

Results of the study based on the ultron-logotron theory (Chung, 2014a) and further review of quantum physics and the Eastern Confucian philosophy reveal remarkable parallels between Confucian philosophy and quantum physics as follows:

1) Confucian philosophy presents the "ultrons" as the building blocks of the physical world, and the ultron-logotron theory proposes the "logotrons" as the building blocks of the consciousness world at quantum levels.

2) There seem to be remarkable parallels between the Confucian philosophy and quantum physics from the ontological perspectives. 
3) Results of the study may suggest existence of eight flavors of quarks and that there would be possibly two more undiscovered flavors of quarks, "light” and “dark” (the author's naming) besides the other six known flavors, probably one positively and one negatively charged, of undetermined mass, and probably extremely short-lived or virtual quarks like $W$ and $Z$ bosons.

4) There could be primary fundamental elementary particles, yin- and yang-“ultrons” in the deeper structure of leptons and quarks.

5) The physical world, the consciousness world and the spiritual world of our universe coexist in superposition and represent the macro-cosmos of the Creator, God. The superpositioned worlds, the macro-cosmos of the Creator of our universe, the Self, seem to be analogous to the physical body, the conscious mind and the inner spiritual self in superposition that represent the micro-cosmos of the co-creator, the self of the humanity.

6) The physical world appears real but essentially not real. The spiritual world is invisible but real. The spiritual world and the human world would unite in the coming kingdom of heaven on Earth where goodness will be boundless according to Jeong Yeok, the Book of Right Change of Confucian philosophy as the Bible predicts it.

7) Virtual particles are in essence virtual logotrons (information) that are the archetype of real particles. Annihilation and creation of ultrons (matter) are secondary to action of underlying virtual logotrons in the Cosmic Consciousness (mind). This seems to suggest that the ultron-logotron theory possibly leads to the Theory of Everything (ToE).

\subsection{Leptons}

The most fundamental building blocks of Nature are currently leptons and quarks (Hawking, 1996, 2001; Best, 2015; Morfin, 2012). There are two kinds of leptons: one of charged electrons and positrons, and one of neutral neutrinos.

\subsubsection{Neutrinos}

Neutrinos are created in the decay of subatomic particles in the sun, galaxy, supernovas, nuclear reactors, particle accelerator laboratories (such as Super-Kamiokande in Japan, IMB in United States) and, cosmic rays in atmosphere (Murayama, 2002). The first neutrino was detected by Cowan and Reines in 1956 (Riordan, 1987 ; Morfin, 2012). Neutrinos are the lightest particles and the least understood of the fundamental particles (Murayama, 2002). Neutrinos have oscillation of three favor types, electron-neutrino, muon-neutrino and tau-neutrino, and are like electrons but have no electric charge. In this study, the symbol of $\sqrt{ }$ and the term of neutrino mean the electron-neutrino.

\subsubsection{Electrons and Positrons}

Electron-positron annihilation occurs when an electron $\left(e^{-}\right)$and positron $\left(e^{+}\right)$collide, resulting in the annihilation of electron and positron, and creation of photons (Dirac, 2014; Wikipedia, 2014a, 2015b).

Electrons are subject to electromagnetism, gravitation and weak nuclear force but not to strong nuclear force.

\subsection{Quarks}

Quarks form composite particles called hadrons of baryons and mesons. Light quarks include up, down and strange quarks. Up and down quarks are the lowest masses of quarks and generally stable and the most common in the universe, being in the atom nucleus and having electric charge of $+2 / 3 e$ or $-1 / 3 e$. A flavor transformation occurs in beta or inverse beta decay between neutron and proton, emitting leptons (Best, 2015; Wikipedia, 2013).

\subsection{String Theory}

In string theory, the point-like particles are replaced by one-dimensional objects called strings that are fundamental building blocks of matter. Different particles arise from different quantum states of strings. String theory incorporates gravity resulting from specific oscillation of strings. One mode of oscillation corresponds to a massless particle such as graviton. Open and closed strings are postulated to be two fundamental elementary particles.

Superstring theories attempt to explain all of the particles and fundamental forces of Nature. M-theory unites 
five superstring theories and is 11-dimensional (Kaku, 1999).

In particle physics, research articles and publications describe ingenious behavior and properties of strings such as oscillation, movements, spin, coupling, interaction, scattering, quantum field, QED, QCD, brane, dimension, compactification, symmetry, propagation, black hole etc. with mathematical descriptions (Best, 2015). There seem to be no set of a defining equation of motion of strings (Kaku, 1999; Louis, Mohaupt, \& Theisen, 2007, Caesar, 2014; Best, 2015).

The deeper structure of the fundamental elementary particles, leptons and quarks is not clearly known in particle physics. There seem to be, to my knowledge, no articles in the literature that clearly elucidate the deeper structure of leptons and quarks.

In this study, findings and discoveries in modern quantum physics are reviewed (Hawking, 1996, 2001; Nichol, 2006; Bohm, 2006; Kaku, 1994, 1999; Penrose, Hameroff, \& Kak, 2011; Planck Collaboration, 2014; Wikipedia, 2015b; Caesar, 2014; Riordan, 1987; Best, 2015; Morfin, 2012). Possible deeper structures of leptons and quarks are investigated and an attempt is made to determine the deeper structures of these particles such as composition, mass and electric charge on the basis of reported data in particle physics from the perspective of the author’s hypothesis of the "ultron”-“logotron” theory (Chung, 2014a, 2014b, 2015).

\section{Results}

Possible deeper structures of leptons and quarks are investigated on the basis of the two following assumptions speculated from the perspective of the author's hypothesis of the ultron-logotron theory:

1) The ultron is the building blocks of matter of all particles of the universe.

2) The neutrino is the smallest and electrically neutral particle among leptons that is a composite of an equal number of yin-ultron $\left(U^{\succ}\right)$ and yang-ultron $\left(U^{+}\right)$. In this study, a neutrino is assumed to be a composite of a yin-ultron and a yang-ultron.

\subsection{Ultron Mass, $\left(m_{u}\right)$}

$m_{u}$ of yin- and yang-ultron is a half of a neutrino mass of $0.320 \pm 0.081 \mathrm{eV}$ (Planck Collaboration, 2014; NIST, 2010, Wikipedia, 2015b).

$$
m_{u}=0.16 \mathrm{eV}\left(1 / 2 \text { of } 9.10938291 \times 10^{-31} \mathrm{~kg}\right)
$$

\subsection{Ultron Size}

Ultron length is postulated to be close to and less than Planck length of $1.616199 \times 10^{-35} \mathrm{~m}$ (Riordan, 1987; Wikipedia, 2015a).

\subsection{Total Number $(N)$ of Ultron within a Lepton, Neutrino, Electron and Positron}

$$
\text { Neutrino } N=2
$$

$N$ is obtained from calculation of electron or positron mass, $m_{e^{-}}$or $m_{e^{+}}$reported as $0.510998928 \mathrm{MeV}$ (or $\left.9.10938291 \times 10^{-31} \mathrm{~kg}\right)($ NIST, 2010) divided by ultron mass as follows:

$$
\text { Electron } N=m_{e^{-}} / m_{u}=0.510998928 \times 10^{6} \mathrm{eV} / 0.16 \mathrm{eV}=3.19 \times 10^{6}
$$

Positron $N$ is same as Electron $N, 3.19 \times 10^{6}$.

\subsection{Total Number $(N)$ of Ultrons within Light Quarks, up, down and Strange}

$\mathrm{N}$ is determined likewise from calculation of up, down or strange mass reported as $m_{u}=2.3(1.8-3.0), m_{d}=4.8$ (4.5 - 5.3), $\mathrm{m}_{\mathrm{s}}=95$ (90 - 100) MeV divided by ultron mass (Beringer et al., 2012).

$$
\begin{gathered}
N(\text { up quark })=14.37 \times 10^{6} \\
N(\text { down quark })=30 \times 10^{6} \\
N(\text { strange quark })=593.75 \times 10^{6}
\end{gathered}
$$




\subsection{Number of Yin- and Yang-Ultron in Leptons}

If yin-ultrons are assumed to be present outside and inside $k$ number of neutrinos in an electrons. Then number of yin-ultron in an electron $=3.19 \times 10^{6}-k$; number of yang-ultron in it is $k$; $k$ value could be 0 or integer $k<$ $3.19 \times 10^{6}$.

Number of yang-ulton in a positron is likewise $3.19 \times 10^{6}-k$; number of yin-ultron is $k$ in a positron.

\subsection{Electric Charge of Yin- and Yang-Ultron}

Both electric charge of yin-ultron and yang-ultron is calculated from the reported electric charge of electron ( $e$ ) and positron $\left(e^{+}\right)$(NIST, 2010) as follows:

$$
\begin{aligned}
e^{-} & =-1.602176565 \times 10^{-19} \mathrm{C} \\
e^{+} & =1.602176565 \times 10^{-19} \mathrm{C}
\end{aligned}
$$

C denotes coulomb.

If $k$ value is equal to zero (no neutrinos), then electric charge of yin-ultron is

Electric charge of $U^{-}=-1.602176565 \times 10^{-19} \mathrm{C} / 3.19 \times 10^{6}=-5.02249707 \times 10^{-26} \mathrm{C}$

Electric charge of $U^{+}=5.02249707 \times 10^{-26} \mathrm{C}$

If $k$ value is not zero, then

$$
\begin{aligned}
& \text { Electric charge of } U^{-}=-1.602176565 \times 10^{-19} \mathrm{C} /\left(3.19 \times 10^{6}-2 k\right) \\
& \text { Electric charge of } U^{+}=1.602176565 \times 10^{-19} \mathrm{C} /\left(3.19 \times 10^{6}-2 k\right) .
\end{aligned}
$$

\subsection{Number of Yin- or Yang-Ultron within Light Quarks, up, down and Strange}

If $w_{u}, w_{d}$ and $w_{s}$ numbers of neutrinos are assumed to be present in an up, a down or a strange quark, respectively, and electric charge of light quarks is:

Electric charge of up quark $=+2 / 3 e$.

Electric charge of down quark $=-1 / 3 e$.

Electric charge of strange quark $=-1 / 3 e$.

Then the number $\left(n_{\text {uyang }}\right)$ of yang-ultron in an up quark would be derived by the following equation:

$$
n_{\text {uyang }}=(2 / 3) \times\left(1.602176565 \times 10^{-19} \mathrm{C}\right) /\left[\left(1.602176565 \times 10^{-19} \mathrm{C}\right) /\left(3.19 \times 10^{6}-2 k\right)\right]+w_{u} .
$$

The number $\left(n_{\text {uyin }}\right)$ of yin-ultron in an up quark would be

$$
n_{\text {uyin }}=w_{u} \text {. }
$$

The number $\left(n_{\text {dyin }}\right)$ of yin-ultron in a down quark would be likewise

$$
n_{\text {dyin }}=(1 / 3) \times\left(-1.602176565 \times 10^{-19} \mathrm{C}\right) /\left[-1.602176565 \times 10^{-19} \mathrm{C} /\left(3.19 \times 10^{6}-2 k\right)\right]+w_{d} \text {. }
$$

The number $\left(n_{\text {dyang }}\right)$ of yang-ultron in a down quark would be

$$
n_{\text {dyang }}=w_{d} .
$$

The number $\left(n_{\text {syin }}\right)$ of yin-ultron in a strange quark would likewise

$$
n_{\text {syin }}=(1 / 3) \times\left(-1.602176565 \times 10^{-19} \mathrm{C}\right) /\left[-1.602176565 \times 10^{-19} \mathrm{C} /\left(3.19 \times 10^{6}-2 k\right)\right]+w_{s} .
$$

The number $\left(n_{\text {syang }}\right)$ of yang-ultron in a strange quark would be likewise

$$
n_{\text {syang }}=w_{s} \text {. }
$$

\section{Discussion}

Superstring theory is a theory unifying bosons (force-carrying particles) and fermions (leptons and quarks), indicating mutual transformation, fermion to boson; boson to fermion. 


$$
\begin{gathered}
\text { fermion } \rightleftarrows \text { boson } \\
\sqrt{ }+p^{+} \rightarrow n^{0}+e^{+} \\
\sqrt{ }+n^{0} \rightarrow p^{+}+e^{-} \\
\text {Energy }(>1.8 \mathrm{MeV})+p^{+} \rightarrow n^{0}+e^{+}+\sqrt{ } \\
{ }^{14} \mathrm{C} \rightarrow{ }^{14} \mathrm{~N}+e^{-}+\sqrt{ } \\
\sqrt{ }+{ }^{37} \mathrm{Cl} \rightleftarrows{ }^{37} \mathrm{Ar}+e^{-}
\end{gathered}
$$

The above experimental results in beta and inverse beta reactions are suggestive of evidence of yet unknown, more fundamental building blocks as their common building blocks of particles in their deeper structure of leptons and quarks, and probably further that the common building blocks might be the ultrons proposed in the ultron-logotron theory. Neutrinos might be composed of a yin- and a yang-ultron (Morfin, 2012; Best, 2015).

In string theory, the extremely small size of Planck length is expected to be close to the string length (Louis et al, 2007; Wikipedia, 2015a). The ultron length is postulated to be close to and less than the Planck length (Riordan, 1987).

In the ultron-logotron theory, a point-like region of space has infinite depth as suggested by the author's "mononary" system of mathematics (Chung, 2015) that seems to be analogous to compactifiation in string theory that allows extra dimensions (5, 10, 11 to 26 dimensions) (Louis et al., 2007).

The ultron is postulated to move and oscillate in various ways like the string does in string theory, and would give rise to different quantum states. Logotrons are also postulated to exist in superposition to ultrons.

Logotrons are virtual particles in Cosmic Consciousness world that is inferred to be timeless and nonlocal, and dimensionless or of zero dimension to infinite dimension as suggested by the author's "mononary" system of mathematics (Chung, 2015). Extra dimensions, 5, 10-dimension of superstring theory, and 11-dimenion of M-theory seem to be possibly understandable. Virtual particles of matter in quantum physics seem to be in essence virtual logotron particles. Virtual particles cannot be observed directly, only when a physical particle is formed that we can observe indirectly. Photons of electromagnetic wave are observed by detecting with physical devices. However, graviton or gluon of virtual, force-carrying particles is observable only through their effect in the physical world. Logotrons are also similarly cognized through their effects.

For the strong nuclear force, gluons clump together into tubes when linking quarks to quarks or to anti-quarks. For the weak nuclear force, photons spherically surround electrons, forming a spherically symmetric shell and responsible for nuclear decay (Best, 2015).

Different quantum states and movements (oscillation) of the ultron-logotron composites give rise to different physical forces, electromagnetism, weak and strong nuclear forces and gravitational force with maximum speed of light of transmission in the physical world, and mental force with instantaneous speed of transmission in the consciousness world. In string theory, different modes of string oscillation give rise to different physical forces (Best, 2015).

All particles of the universe mutually exchange physical force and mental force particles and respond in various degrees. In this respect, everything of Nature is interrelated and interconnected (Capra, 1999; Chung, 2014a, 2014b, 2015).

Four virtual physical forces of Nature, electromagnetic force, weak and strong nuclear forces and gravitational force are postulated to be different manifestations of mental-force carrying "mentalon" particles associated with ultron-logotrons in superposition from the perspective of the hypothesis of the ultron-logotron theory (Chung, 2014a, 2014b, 2015). Logotrons are inferred to be the archetype of ultrons (real particles of matter) and the source of forces. The ultron-logotron theory might lead to the Theory of Everything.

The relationship between yin-ultron and yang-ultron is not like electron and positron, ie, matter and antimatter. The relationship among yin-ultron, yang-ultron and neutrino seem to be analogous to that among electron, proton and neutron. When yin- and yang-ultrons meet, there would be no annihilation but a neutrino composite. When high-energy electron and positron collide, they annihilate and produce pure high energy that can give birth to other particles and antiparticles such as muons, mesons or quarks with subsequent hadronization. According to quantum field theory, all particles can be transmuted into other particles, and they can be created from energy and can vanish into energy (Riordan, 1987; Capra, 1999; Best, 2015). 


$$
\begin{gathered}
e^{-}+e^{+} \rightarrow \gamma \rightarrow \text { hadrons } \\
e^{-}+e^{+} \rightarrow q+\text { anti- } q \rightarrow \text { hadrons }
\end{gathered}
$$

The results of high-energy electron-positron collision and neutrino scattering experiments at SLAC, SPEAR, HEPL, NAL, PETRA, Fermilab, CERN, DESY, etc. suggest existence of elementary particles, possibly yin- and yang-ultrons in the deeper structure of leptons and quarks. The existence of quark is known but quarks are never directly observed or found in isolation; they can be found only within hadrons (Best, 2015; Riordan, 1987; Cabbibo \& Gatto, 1961; Kaye, 1983; Wikipedia, 2015a). It is inferred that ultrons and neutrinos are arrayed in specific, geometrical features in the deeper structure of leptons and quarks. Ultrons may possibly hide in scattering debris in the detector in particle collision experiments and in cosmic ray. Abdus Salam (Riodan, 1987) coined the word preon for the imaginary constituents of quarks and leptons that seems to be analogous to the ultron.

There seems to be possible parallels between the theory of the Eightfold Way of Gell-Mann, Nobel laureate, in quantum physics (the word comes from Buddha's teaching of the path to Nirvana) and the Eight Trigram of Jeong Yeok in Confucian philosophy (Riordan, 1987; Chung, 2014b, 2015; Rosner, 2002; Sener \& Schulten, 2015). The Eightfold Way (octets) and triplets (three quarks) are analogous to the Eight Trigram of Jeong Yeok and trigram in Confucian philosophy.

Further research would be needed for verification of the results presented in this study and the hypothesis of the ultron-logotron theory.

\section{Conclusion}

Findings and discoveries in modern quantum physics and theoretical physics regarding fundamental elementary particles are reviewed in this study. The following hypothesis related to possible deeper structures of leptons and light quarks, and some properties of ultrons are proposed on the basis of reported data in particle physics and the author’s hypothesis of the "ulton”-“logotron” theory (Chung, 2014a, 2014b, 2015).

1) A neutrino $(\sqrt{ })$ is composed of an equal number of yin-ultron $(U)$ and yang-ultron $\left(U^{+}\right)$. In this study, it is postulated to be composite of one yin-ultron and one yang-ultron.

2) Yin- and yang-ultron mass is $0.16 \mathrm{eV}$.

3) Electric charge of yin-ultron, $U$ and yang-ultron, $U^{+}$:

Electric charge of yin-ultron $=-1.602176565 \times 10^{-19} \mathrm{C} /\left(3.19 \times 10^{6}-2 k\right)$.

Electric charge of yang-ultron $=1.602176565 \times 10^{-19} \mathrm{C} /\left(3.19 \times 10^{6}-2 k\right)$.

$\mathrm{C}$ denotes coulomb and $k$ a number of neutrinos in a lepton.

4) Ultron size is close to and less than Planck length of $1.616199 \times 10^{-35} \mathrm{~m}$.

5) A possible total number $(N)$ of ultrons in the deeper structure of leptons and light quarks are as follows:

Neutrino $N=2$.

Electron $N=3.19 \times 10^{6}$.

Positron $N=3.19 \times 10^{6}$.

Up quark $N=14.37 \times 10^{6}$.

Down quark $N=30 \times 10^{6}$.

Strange quark $N=593.75 \times 10^{6}$.

6) Number $(N)$ of yin-and yang-ultrons in the deeper structure of leptons and light quarks are presented in this study (see Equations (2), (3), (9.1) to (9.6)).

Further experimental research in particle physics would be needed for verification of the above results and the hypothesis regarding possible deeper structures of leptons and quarks proposed from the perspective of the ultron-logotron theory.

\section{References}

Aczel, A. D. (2003). Entanglement. New York: Plume.

Beringer et al. (Particle Data Group) (2012). Light Quarks (u, d, s). http://pdg.lbl.gov/2012/listings/rpp2012=list-light-quarks.pdf

Best, B. (2015). The Standard Model of Particle Physics. http://www.benbest.com/science/standard.html

Bohm, D. (2006). Wholeness and the Implicate Order. New York: Routledge. 
Cabibbo, N., \& Gatto, R. (1961). Electron-Positron Colliding Beam Experiments. Physical Review, 124, 1577-1598. http://dx.doi.org/10.1103/PhysRev.124.1577

Caesar, C. (2014). On the Substructure of the Electron. http://www.ccaesar.com/index.html

Capra, F. (1999). The Tao of Physics. Boston: Shambhala.

Chung, S. J. (1960). Studies on a Mathematical Relationship between Stress and Respose in Biological Phenomena. Journal of the National Academy of Sciences, Republic of Korea, 2, 115-162.

Chung, S. J. (1995). Formulas Expressing Life Expectancy, Survival Probability and Death Rate in Life Table at Various Ages in US Adults. International Journal of Biomedical Computing, 39, 209-217. http://dx.doi.org/10.1016/0020-7101(94)01068-C

Chung, S. J. (2007). Computer-Assisted Predictive Formulas Expressing Survival Probability and Life Expectancy in US Adults, Men and Women, 2001. Computer Methods and Programs in Biomedicine, 86, 197-209. http://dx.doi.org/10.1016/j.cmpb.2007.02.009

Chung, S. J. (2009). Seeking a New World: A New Philosophy of Confucius and Kim Hang. Bloomington, IN: iUniverse.

Chung, S. J. (2010). The Book of Right Change, Jeong Yeok 正易: A New Philosophy of Asia. Bloomington, IN: iUniverse.

Chung, S. J. (2012). The Science of Self, Mind and Body. Open Journal of Philosophy, 2, 171-178. http://dx.doi.org/10.4236/ojpp.2012.23026

Chung, S. J. (2013). Mathematical Relationship of “Probacent”-Probability Equation among Exogenous Stressor, Stress and Response in Biological Phenomena. International Journal of Education and Research, 1, 1-32. http://www.ijern.com/September-2013.php

Chung, S. J. (2014a). Self and Consciousness: Mind and Matter. International Journal of Education and Research, 2, 1-28. http://www.ijern.com/journal/March-2014/35.pdf

Chung, S. J. (2014b). Parallels between Confucian Philosophy and Quantum Physics. Open Journal of Philosophy, 4, 192206. http://scirp.org/journal/ojpp

Chung, S. J. (2015). On the Evolution of Humanity: View from the "Ultron”-“Logotron” Theory. International Journal of Humanities and Social Science, 5, 50-63. http://www.ijhssnet.com/index.php/journal/index/2920

Close, F. (2011). The Infinity Puzzle. New York: Basic Books.

Dirac, P. (2014). Dirac Equation. http://en.wikipedia.org/wiki/Dirac_equation

Greene, J. R., Sommerville, R. B., Nystrom, L. E., Darley, J. M., \& Cohen, J. D. (2001). An fMRI Investigation of Emotional Regulation in Moral Judgment. Science, 293, 2150-2108. http://dx.doi.org/10.1126/science.1062872

Griffiths, D. J. (2005). Introduction to Quantum Mechanics. Noida: Pearson.

Gruenberg, L. A. (1959). Defining Moment: The Korean War. Detroit, MI: Graphiccs.

Gusnard, D. A. (2009). Basic Science of Self. In B. J. Sadock, V. G. Sadock, \& P. Ruiz (Eds.), Kaplan and Sa dock’s Comprehensive Textbook of Psychiatry (pp. 353-363). New York: Lippincott Williams \& Wilkins.

Hameroff, S. R. (2013). Quantum Computation in Brain Microtubules? The Penrose-Hameroff “Orch OR” Model of Consciousness. Philosophical Transactions of the Royal Society of London A, 356, 1869-1896.

Hawking, S. (1996). A Brief History of Time. New York: Bantam Books.

Hawking, S. (2001). The Illustrated a Brief History of Time. New York: Bantam Books.

Joseph, R. (2011a). Dreams and Hallucinations: Lifting Veil to Multiple Perceptual Realities. In R. Penrose, S. Hameroff, \& S. Kak (Eds.), Consciousness and the Universe (pp. 516-550). Cambridge, MA: Cosmology Science Publishers.

Kafatos, M., Tanzi, R. E., \& Chopra, D. (2011). How Consciousness Becomes the Physical Universe. In R. Penrose, S. Hameroff, \& S. Kak (Eds.), Consciousness and the Universe (pp.1119-1129). Cambridge, MA: Cosmology Science Publishers.

Kaku, M. (1994). Hyperspace: A Scientific Odyssey through Parallel Universe, Time Warps, and the Tenth Dimension. New York: Doubleday.

Kaku, M. (1999). Introduction to Superstrings and M-Theory (2nd ed.). New York: Springer. http://dx.doi.org/10.1007/978-1-4612-0543-2

Kaye, S. (1983). Quark Flavor Identification in Electron-Positron Annihilation. http://www.slac/ac.stanford.edu/cgi-wrap/getdoc/slac-r-262.pdf

Kim, H. (1885). Jeong Yeok, 金恒, 正易, 정역. The Original Chinese Text with the Text Translated in Korean by Jeong Ho Yi. Seoul: The Asian Culture Press.

Laszlo, E. (2007). Science and the Akashic Field. Rochester, VT: Inner Traditions. 
Laszlo, E. (2008). Quantum Shift in the Global Brain. Rochester, VT: Inner Traditions.

Louis, J., Mohaupt, T., \& Thelsen, S. (2007). String Theory: An Overview. Lecture Notes Physics, 721, $289-323$. http://dx.doi.org/10.1007/978-3-540-71117-9_12

McGonigal, K. (2012). The Willpower Instinct. New York: Avery.

Miller, E. K., \& Cohen, J. D. (2001). An Integrative Theory of Prefrontal Cortex Function. Annual Review of Neuroscience, 24, 167-202. http://dx.doi.org/10.1146/annurev.neuro.24.1.167

Mitchell, E. D., \& Staretz, R. (2011). The Quantum Hologram and the Nature of Consciousness. In: R. Penrose, S. Hameroff, \& S. Kak (Eds.), Consciousness and the Universe (pp. 18-19). Cambridge, MA: Cosmology Science Publishers.

Morfin, J. G. (2012). Neutrino Physics: What's a Neutrino and How Does It Interact? http://users.physics.psu.edu/ cteq/schools/summer09

Murayama, H. (2002). The Origin of Neutrino Mass. Physics Today, May 2002, 35-39.

Nichol, L. (2006). The Essential David Bohm. New York: Routledge.

NIST (2010). Fundamental Physical Constants. http://physics.nist.gov/cgi-bin/cuu/Value?me2mev

Ochsner, K. N., Bunge, S. A., Gross, J. J., \& Gabrieli, J. D. (2002). Rethinking Feelings: An fMRI Study of the Cognitive Regulation of Emotion. Journal of Cognitive Neuroscience, 14, 1215-1229. http://dx.doi.org/10.1162/089892902760807212

Penrose, R., Hameroff, S., \& Kak, S. (2011). Consciousness and the Universe. Cambridge, MA: Cosmology Science Publishers.

Penrose, R. (2007). The Road to Reality: A Complete Guide to the Laws of the Universe. New York: Vintage Books.

Penrose, R., \& Hameroff, S. (2011). Consciousness in the Universe: Neuroscience, Quantum Space-Time Geometry and Orch OR Theory. In R. Penrose, S. Hameroff, H. P. Stapp, \& D. Chopra (Eds.), Consciousness and the Universe: Quantum Physics, Evolution, Brain and Mind. Cambridge, MA: Cosmology Science Publishers.

Planck Collaboration (2014). http://arxiv.org/abs/1303.5076. http://en.wikipedia.org/wiki/Neutrino.

Riordan, M. (1987). The Hunting of the Quark: A True Story of Modern Physics. New York: Simon \& Schuster, Inc.

Rosner, J. L. (2002). The Eightfold Way. In J. S. Rigden, J. Bagg, \& R. H. Stuewer (Eds.), Macmillan Encyclopedia of Physics, Supplement: Elementary Particle Physics. New York: Macmillan Reference USA.

Schwartz, J. M. (1999). A Role for Volition and Attention in Generation of New Brain Circuitry: Toward Neurobiology of Mental Force. Journal of Consciousness Study, 6, 115-142.

Schwartz, J., Stapp, H. P., \& Beaureguard, M. (2005). Quantum Physics in Neuroscience and Psychology: A Neurophysical Model of Mind/Brain Interaction. Philosophical Transactions of the Royal Society B, 360, 1309-1327.

http://dx.doi.org/10.1098/rstb.2004.1598

Sener, M., \& Schulten, K. (2015). Symmetries in Physics: Isopin and the Eightfold Way. http://www.ks.uiuc.edu/Services/Class/PHYS480/qm_PDF/chp12.pdf

Stapp, H. P. (1999). Attention, Intention, and Will in Quantum Physics. Journal of Consciousness Study, 6, 143-164.

Stapp, H. P. (2011). Mindful Universe. http://www.thedivineconspiracy.org/25256H.pdf http://dx.doi.org/10.1007/978-3-642-18076-7

Suplee, C. (1999). Physics in 20th Century. New York: Harry N. Abrams, Inc.

Tong, D. (2009). String Theory. http://www.damtp.cam.ac.uk/user/tong/string/string.pdf

Watson, A. (1997). Quantum Spookiness Wins, Einstein Loses in Photon Test. Science, 277, 481. http://dx.doi.org/10.1126/science.277.5325.481

Wikipedia (2013) Quark. http://en.wikipedia.org/index.php?title=Quark\&oldid=574208413

Wikipedia (2014a). Electron-Positron Annihilation. http://en.wikipedia.org/wiki/Electron-positron annihilatiion

Wikipedia (2014b). Chlorine-37. http://en.wikipedia.org/index.php?title=Chlorine-37\&oldid=599739482

Wikipedia (2015a). String Theory. http://en.wikipedia.org/wiki/String_theory

Wikipedia (2015b). Electron-Positron Annihilation into Quark-Antiquark. http://hepwww.rl.ac.uk/OpenDays98/Century\%20of\%electrons/quark3.html

William, R. (1967). The I Ching or the Book of Changes. London: Rutledge \& Kegan Paul.

Yi, J. H. (1992). The Third Yeok Hak, 第三의易學 (The Author's Note: Jeong Yeok as The Third Yeok) (Text in Korean). Seoul: The Asian Culture Press. 